Volume etc

\title{
Hearing the voices of general staff: A Delphi study of the contributions of general staff to student outcomes $^{\dagger}$
}

\author{
Carroll Graham*
}

Faculty of Arts and Social Sciences, University of Technology, Sydney, Sydney, Australia

\begin{abstract}
The key resource for universities is their academic and general staff; however, little attention has been paid, by government, researchers and even the universities in which they work, to the work of general staff. Yet general staff have comprised more than half the workforce in Australian universities since 1996, and a more rigorous understanding of the contribution of general staff towards the strategic goals of their institutions has the potential to enhance their institutions' organisational sustainability. While universities have multiple and diverse stakeholders, students occupy the key stakeholder role in a university's core business of learning and teaching. Consequently, the interaction of general staff with students has potential to impact on the sustainability of an institution. This paper describes a preliminary study into how general staff contribute to student outcomes. A meta-study by Prebble et al. (2004) derived 13 propositions for support of student outcomes that focussed on the contribution by academic staff, and Middleton (2006) subsequently surmised that general staff are also central to those outcomes. This study uses the Delphi method to test Middleton's (2006) assertion by engaging general staff in ranking the propositions in terms of the contribution of general staff to student outcomes. This paper concludes by discussing implications for future research.
\end{abstract}

Keywords: general staff; higher education; student outcomes; Delphi Method

\section{Introduction}

Over the last twenty years, there has been growing concern about accountability in higher education in Australia (Adams, 1998). Public and government concern has coincided with or been triggered by the massification of higher education, the move from the binary system to the Unified National System, economic rationalism and the consequent changes to student profiles and funding arrangements (Adams, 1998; Ng, Heskin, \& Sharma, 1993). There have been consequent calls for greater effectiveness and efficiency in higher education and the concomitant need for measurement. One measure of an institution's performance and accountability has been identified by a number of commentators (Clark cited in Elford, 1996; $\mathrm{Ng}$, et al., 1993) as student outcomes assessment.

Conway (2000) asserts that university administrators are, as a group, ignored by government, by universities themselves, and by academics with whom administrators work. Similarly, Szekeres (2004) laments the lack of literature concerning general staff (and, in particular, administrative staff) and refers

\footnotetext{
${ }^{\dagger}$ An earlier version of this paper was presented at [name of conference deleted to maintain the integrity of the review process]

*Email: carroll.graham@uts.edu.au
} 
to general staff as "the invisible workers" (p. 7). General staff have comprised more than half the workforce in Australian universities since 1996 (DEEWR, 2009) and the responsibilities undertaken by this group are diverse, comprehensive and considerable. Such a large workforce, more than 54,800 in 2008 (DEEWR, 2009) invites a more rigorous understanding of the work undertaken, and the contribution made by general staff to the strategic goals of their institutions.

This paper is concerned with the work undertaken by general staff in Australian universities, and focusses on the question of how general staff contribute to student outcomes. In a review of 146 international studies, Prebble et al. (2004) derived 13 propositions for student support (Table 1) that were found to enhance student outcomes in terms of retention, persistence and achievement. Although the study by Prebble et al. (2004) focussed on the contribution to student outcomes by academic staff, Middleton (2006) speculated that general staff are also central to those outcomes. The current study takes the somewhat unusual approach of asking general staff about their work. In particular, this study gives voice to general staff at [name deleted to maintain the integrity of the review process] in relation to the importance of their contributions to student outcomes, using the propositions developed by Prebble et al. (2004) as a framework.

Table 1: Institutional behaviours that support student outcomes ${ }^{\text {a }}$

\section{Student Support Propositions Description}

1. Institutional behaviours, environments and Students' enquiries are dealt with promptly, processes are welcoming and efficient

2. The institution provides opportunities for students to establish social networks knowledgeably and with a friendly manner

Student clubs, societies and activities are supported, and facilities and events are provided to support socialisation

3. Academic counselling and pre-enrolment advice Students are provided with high quality advice and are readily available to ensure students enrol in appropriate programs

4. Lecturers are approachable and accessible inside and outside class times for academic discussions

5. Students experience good quality teaching and manageable workloads information concerning program choices, and links are established with secondary schools

Students benefit from regular and meaningful formal and informal contact with academics, particularly when a learning community is developed

The quality and teaching methodologies can have an impact on student outcomes, as can a manageable workload

6. Orientation and induction programs are provided to facilitate both social and academic integration

Both academic orientation and general orientation programs can improve student outcomes

7. Students working in academic learning communities have good outcomes

The deliberate use and facilitation of learning communities has a positive impact on student outcomes

8. A comprehensive range of institutional services and facilities is available

9. Supplemental instruction is provided

10.Peer tutoring and mentoring services are provided

Student outcomes are improved by the provision of services and facilities that support both the social and academic integration of students

Academic support programs in programs that students find difficult improve student outcomes

Students benefit from well-designed and well-run peer tutoring and mentoring programs 
11. The institution ensures there is an absence of Students need to feel safe, valued and respected discrimination on campus, so students feel valued, fairly treated and safe

12. Institutional processes cater for diversity of Students have different learning styles, which learning preferences need to be accommodated

13. The institutional culture, social and academic, The diverse backgrounds of students should be welcomes diverse cultural capital and adapts to diverse students' needs

${ }^{a}$ From Prebble et al. (2004)

\section{The study}

\section{Design of the study and research method}

The aim of this study was to elicit the views of a selection of general staff about their work in relation to student outcomes. In order to do this, it was decided to draw on previous meta-study research as a basis and framework for the current study. Accordingly, a modification of the Schmidt Delphi method (R. C. Schmidt, 1997) was used to develop a rank order for the 13 propositions for student support developed by Prebble et al. (2004) in terms of how general staff perceive their contributions to each proposition. The Schmidt Delphi method for ranking items involves three phases: a brainstorming phase to develop a list of issues; a narrowing down phase to pare the list of issues; and a ranking phase to order the remaining items (R. Schmidt, Lyytinen, Keil, \& Cule, 2001). For the purposes of this study, the meta-study by Prebble et al. (2004) and the associated development of the propositions are considered to be the first two phases.

The Delphi study was located at one site only: [name deleted to maintain the integrity of the review process]. This site was chosen due to the representative nature of [name deleted to maintain the integrity of the review process] in terms of its provenance, location, size and diversity: it was established as a public university from a previous institution some 20 years ago (as have half Australia's universities); it is located in [name deleted to maintain the integrity of the review process] (which has more universities than any other state of Australia); with 32,000 students it is a medium-to-large university; it is sited in a capital city (as are most universities in Australia); with 23\% international students, [name deleted to maintain the integrity of the review process] is close to the overall Australian figure for international students of 26.5\% (DEEWR, 2008; Marginson \& Considine, 2000).

\section{The Delphi method}

The Delphi method is a group process that gathers and synthesises the opinions of several individuals, considered to be experts in the field of study, to improve the quality of decision-making (Delbecq, Van de Ven, \& Gustafson, 1975). Essentially, the Delphi method is a series of questionnaire rounds, which is interspersed with controlled feedback to the participants that is based on the results of the previous round. The purpose of this method is to create group consensus from individual opinions (Hasson, Keeney, \& McKenna, 2000). Schmidt (1997) adapted the Delphi method for the ranking of key issues. The Delphi method does not require the experts to meet physically, thereby reducing the logistical constraints of the study and it may involve the key stakeholders. As such, the Delphi method is highly suitable for practice-based research. 


\section{Composition of the panel}

Choosing appropriate experts is an important aspect of Delphi studies (Adler \& Ziglio, 1996; Delbecq, et al., 1975; Duffield, 1993; Okoli \& Pawlowski, 2004), and there are two key aspects to this: panel size and knowledge of the panellists (Powell, 2003). A single expert is unlikely to have had personal experience of all the behaviours described by the propositions, and therefore the use of a ranking-style Delphi study, using a process of iterative, controlled feedback will provide a broader experience base from which to develop consensus (R. Schmidt, et al., 2001). Typically, panellists should be chosen who are representative of their profession, have power to implement the findings or who are unlikely to be challenged as experts (Fink, Kosecoff, Chassin, \& Brook, 1984). However, the number of experts required for a panel is not large, with 10 to 18 being considered suitable (Paliwoda, cited in Okoli \& Pawlowski, 2004).

Delphi study panellists should meet four overarching criteria: i) knowledge and experience with the issues under study; ii) the capacity and willingness to contribute to the investigation; iii) sufficient time or the study; and iv) adequate communication skills (Adler \& Ziglio, 1996). Accordingly, criterion sampling was used to select potential participants on the basis of skills and attributes that would provide a suitable basis for the panellists to rank the propositions most appropriately. Criteria for knowledge and experience were that panellists had at least 5 years' experience in higher education and, as the study concerns student outcomes, that they work close to the "academic coal face" — that is, general staff located in faculties or schools across a range of roles (Table 2). Approval-in-principle to approach staff was firstly obtained from faculty managers, and then a list of 105 names of staff meeting these criteria were provided by managers in five of the seven faculties. From this list, 44 staff were selected across the range of five faculties and a diverse variety of positions, thereby providing a representative group of experts as recommended by the literature (Powell, 2003). A short outline of the research with a request for participation was then sent to these staff.

Table 2: Criteria for expert selection for Delphi study

\begin{tabular}{lll}
\hline Dimension & Criterion & Example \\
\hline Experience in higher education & Five years or more & \\
Faculty / School & Professional (science-based) & Engineering, IT \\
Faculty / School & Professional (social-science based) & Education, Law \\
Faculty / School & Generalist & Arts, Science \\
Role & Administration & Student adviser \\
Role & Technical & Professional officer \\
Role & Curriculum & Educational designer \\
Role & Non-curriculum & Mentor program manager \\
Role & Marketing / outreach & Faculty / School marketer \\
Role & Managerial & Faculty / School manager \\
\hline
\end{tabular}

Ultimately, 26 panellists, 14 women and 12 men, returned the consent form and participated in the study. This gender balance fits with the overall gender balance at [name deleted to maintain the integrity of the review process] (58\% female and $42 \%$ male). The length of experience in higher education ranged from seven years to 32 years, with an average of 16 years. Typically, the panellists have worked only at [name deleted to maintain the integrity of the review process], although one panellist reported having worked at six universities, with the average number of universities at which panellists have worked being less than 
two. Panellists worked in positions ranging from Higher Education Worker Level 4 to above Level 10, with the average being Level 7. On average, the panellists' highest educational qualification was a bachelor degree. The demographics of the panel are shown in Table 3.

Table 4 indicates the range of faculties and roles included in the panel. In order to preserve confidentiality, the roles have been clustered into three groups: management (administrative and technical); technical (including research and teaching support); and administrative (including academic staff support, marketing/development, student placements, research administration, facilities and finance administration). The largest cluster was the administrative staff, with 15 of the 26 panellists working in some form of administrative role.

Table 3: Demographics of expert panel

\begin{tabular}{llll}
\hline Characteristic & Avg & Min & Max \\
\hline Panellist's experience in higher education (years) & 16.06 & 7 & 32 \\
Number of universities at which panellists have worked & 1.65 & 1 & 6 \\
Panellist's educational level $^{1}$ & BD & HS & DD \\
HEW $^{2}$ level & 7 & 4 & $>10$ \\
\hline
\end{tabular}

1 Education level is the highest level attained by the panellist: HS = high school leaving certificate (or equivalent), $\mathrm{BD}=$ bachelor degree, $\mathrm{DD}=$ doctoral degree

2 HEW (Higher Education Worker) level is the current level of the panellist: 3 panellists indicated that previous roles had been at higher levels

Table 4: Faculties and roles of expert panel

\begin{tabular}{|c|c|c|c|}
\hline \multirow[b]{2}{*}{ Faculty } & \multicolumn{3}{|c|}{ Role (number of panellists) } \\
\hline & Management $^{1}$ & Technical $^{2}$ & Administrative $^{3}$ \\
\hline Faculty of Arts and Social Sciences & 1 & 1 & 3 \\
\hline Faculty of Engineering and IT & 3 & 0 & 4 \\
\hline Faculty of Design, Architecture and Building & 1 & 1 & 1 \\
\hline Faculty of Nursing, Midwifery \& Health & 1 & 0 & 4 \\
\hline Faculty of Science & 1 & 2 & 3 \\
\hline
\end{tabular}

1 Includes administrative and technical management

2 Includes a range of technical roles

${ }^{3}$ Includes a broad range of administrative roles

\section{Data collection and analysis method}

\section{Collection}

The propositions developed by Prebble et al. (2004) were used as a starting point for phase 3 of the Schmidt Delphi method (R. Schmidt, et al., 2001). Typically, three rounds of surveys are conducted (Powell, 2003), at which time consensus is generally reached (R. Schmidt, et al., 2001). Keeney, Hasson and McKenna (2006) note that this is reflected in the literature they examined, where two to four rounds 
were used. It is also noted that panellists can suffer from fatigue as the number of rounds increase (Starkweather, Gelwicks \& Newcomer cited in Keeney, et al., 2006), and McKenna (cited in Keeney, et al., 2006) felt that response exhaustion occurred after two rounds, particularly for busy experts. Accordingly, it was decided to use three ranking rounds for the current study, to balance the desire for consensus with the risk of panel-fatigue and the associated ethical considerations. All three rounds were conducted over a 5-week period during May and June 2009.

In Round 1, 24 panellists were sent an introductory letter with a request to rank the propositions in terms of the importance of the contribution by general staff to each proposition, and a survey form listing, in numerical order, the 13 propositions with their descriptions (Table 1). The survey form also allowed space for open-ended comments concerning the propositions or the contributions made by general staff to student outcomes. The author collected the survey forms, in person, on a nominated date, in order to maximise the return: all 24 panellists completed the survey. Collection of the completed forms by the author in person also allowed for panellists to make additional verbal comments. For Round 2, three of the propositions relating most strongly to academic impact (No. 4, 5 and 12) were culled from the survey form. Background information relating to the derivation of the propositions was provided, along with a summary of the analysis of Round 1, and a letter of explanation. In the analysis summary, the propositions were ordered according to the percentage of panellists ranking each proposition in the top half of the rankings, from highest to lowest. An indication of the level of agreement of the panel, as derived using Kendall's coefficient of concordance (W) (Kendall \& Gibbons, 1990; R. C. Schmidt, 1997), was also provided.

Twenty-six panellists were sent the Round 2 survey, comprising the original 24 as well as two additional panellists for whom the consent form had been received after Round 1 had started. Two of the original panellists had gone on leave and so did not complete the Round 2 survey, and one panellist was unable to complete the survey due to work commitments, resulting in 23 completed Round 2 surveys. For Round 3 , an analysis summary of Round 2 was provided to the 26 panellists, again indicating the level of agreement and the percentage of panellists ranking each proposition in the top half of the rankings, as described above. In addition, the mean rank of each proposition was also provided along with a short commentary on the emerging patterns. In Round 3, all but one of the panellists (who was still on leave) returned the survey, giving 25 completed surveys. Demographic data was collected separately in Round 1 from the original 24 panellists and in Round 3 from the additional two panellists.

\section{Analysis}

Kendall's coefficient of concordance, $W$ (Equation 1), is widely recognised as the best metric for measuring non-parametric rankings (Okoli \& Pawlowski, 2004). The value of $W$ ranges from 0 to 1 (Kendall \& Gibbons, 1990), with a value of 0 indicating no consensus and a value of 1 indicating perfect agreement between the experts (Okoli \& Pawlowski, 2004). Schmidt (1997) developed a guideline to the interpretation of Kendall's $W$ (Table 5), but cautioned that these values should not be applied rigidly.

\section{Equation 1: Calculation of Kendall's coefficient of concordance (W)}

$$
W=\frac{12 S}{m^{2}\left(n^{3}-n\right)-m T}
$$

where:

$S=$ sum of squares of the deviations of the rankings from the mean rankings

$m=$ number of experts

$n=$ number of propositions being ranked (13) 
$T=$ is a correction factor for tied ranks

Table 5: Interpretation of Kendall's coefficient of concordance ${ }^{a}$

\begin{tabular}{lll}
\hline $\boldsymbol{W}$ & Interpretation & Confidence in Rankings \\
\hline 0.1 & Very weak agreement & None \\
0.3 & Weak agreement & Low \\
0.5 & Moderate agreement & Fair \\
0.7 & Strong agreement & High \\
0.9 & Unusually strong agreement & Very high \\
\hline a & From (R. C. Schmidt, 1997) &
\end{tabular}

\section{Results}

The results for Kendall's coefficient of concordance showed increasing agreement over the three rounds. Nevertheless, agreement by Round 3 was no more than "moderate" (Table 6), with the number of panellists in each round varying between 23 and 25 .

Table 6: Kendall's coefficient of concordance and its interpretation for each round

\begin{tabular}{llll}
\hline & Round 1 (n=24) & Round 2 (n=23) & Round 3 (n=25) \\
\hline Kendall's $W$ & 0.29 & 0.34 & 0.47 \\
Interpretation of $W$ & Weak agreement & Weak agreement & Moderate agreement \\
\hline
\end{tabular}

The most highly ranked proposition in all three rounds was the first, namely: Institutional behaviours, environments and processes are welcoming and efficient; that is, students' enquiries are dealt with promptly, knowledgeably and with a friendly manner. This proposition was ranked in the top half of the rankings by all panellists for the first and third rounds, and by all but one panellist in the second round. Other propositions that were consistently ranked highly over all three rounds were numbers 3, 6 and 8 . In addition to the propositions relating strongly to the behaviour of academics, propositions 9,10 and 13 were consistently ranked low. The average ranks of each proposition by the end of Round 3 are shown in Table 7, along with the percentage of panellists ranking each proposition in the top half of the rankings.

Table 7: Ranking of propositions after Round 3

Student Support Propositions $\quad$ Mean Rank $\begin{gathered}\text { Percentage ranking } \\ \text { in top half }\end{gathered}$

1. Institutional behaviours, environments and processes are $\quad 1.48 \quad 100$ welcoming and efficient 
3. Academic counselling and pre-enrolment advice are readily

available to ensure students enrol in appropriate programs

6. Orientation and induction programs are provided to facilitate

both social and academic integration

8. A comprehensive range of institutional services and 4.28 facilities is available

11. The institution ensures there is an absence of discrimination on campus, so students feel valued, fairly treated and safe

7. Students working in academic learning communities have good outcomes

2. The institution provides opportunities for students to establish social networks

9. Supplemental instruction is provided

10. Peer tutoring and mentoring services are provided

13. The institutional culture, social and academic, welcomes diverse cultural capital and adapts to diverse students' needs

4. Lecturers are approachable and accessible inside and outside class times for academic discussions

5. Students experience good quality teaching and manageable workloads

12. Institutional processes cater for diversity of learning preferences

\section{Discussion and implications}

\section{Use of a single site}

Although the characteristics of [name deleted to maintain the integrity of the review process] make it representative of many Australian universities, there are others, notably rural and remote institutions, for which the culture, staff and student characteristics are quite different. Indeed, the characteristics of the student body are central to the design and development of effective student support systems, and within any student body there may be several cohorts of students having different characteristics that require different consideration (Tait, 2000). For example, the support needs of part-time, mature-age, external students differ markedly from those of the recent school-leaver. While it is not possible to make generalisations from the current study, it does raise questions for further study: would a more heterogeneous panel give different results? what would a replication of this study at a different university (or universities) find? what would the results be from a panel that comprised central student support staff, such as careers advisers, equity staff and health professionals?

\section{Limitations of the Prebble propositions}

The report by Prebble et al. used 146 studies, published between 1993 and 2003, to contribute to its findings (2004). Given the rapid changes that have occurred in both higher education and more generally during this period, the relevance of the propositions in 2009 is open to question. In fact, during the Round 1 collection, one of the panellists verbally commented on the lack of propositions relating to 
pedagogical support through technology. Increasingly, learning and teaching is relying on technology for delivery and support of their learning needs (Salaway, Caruso, \& Nelson, 2007), much of which is designed, developed and maintained by general staff. Additional, in-depth study is required to explore these and other issues. Moreover, the study by Prebble et al. (2004) considered only influences on student outcomes in undergraduate tertiary study; other key student cohorts are postgraduate coursework students and higher degree research students. Further investigation would be needed to test the propositions for postgraduate student support.

\section{The silent voice of general staff?}

In collecting the Round 1 survey forms, discussions with the panellists revealed a lack of clarity concerning the ranking criteria. Comments by panellists to the author indicated that, in this round, several panellists had ranked the propositions in order of importance, as they saw it, to student outcomes, rather than in order of the contributions by general staff to the propositions. This lack of clarity was also revealed in comments by two of the panellists in the open-ended section:

This survey is very difficult to do with any accuracy. The propositions are really questions for Academics that teach courses. The order of these is also very difficult to decide. It is like trying to rank fruit - all are good [author's emphasis].

I had to redo my ranking when I understood the requirement of 'general staff contribution to...'.

This divergence of interpretation of the question was reflected in the low level of agreement in the ranking order found for Round 1. In order to clarify the intent of the enquiry, the information letter for subsequent rounds emphasised that the questions related to the contributions made by general staff by including the instruction: "When considering the rank, please ask yourself: 'to which of these propositions do general (support) staff contribute most?" " [emphasis in the original]. As noted earlier, general staff are "the invisible workers" (Szekeres, 2004, p.7) - perhaps it could be argued that general staff are so seldom asked about their work that they almost instinctively answer in terms of the impact for others?

\section{Panel diversity: the voices of general staff}

In keeping with the recommendations from literature, a heterogeneous group, including panel members with varied backgrounds, was selected to engender high quality outcomes (Powell, 2003). However, the wide range of responses, evidenced by the relatively low level of agreement as derived from Kendall's coefficient of concordance, is likely to have arisen from this diversity. This influence was noted by several panellists in their open-ended written comments, as illustrated below:

It's difficult to deal with 'general staff' as one homogeneous group. The importance of the various propositions overleaf varies according to which general staff are involved eg. technical staff have an impact more akin to academic staff so the "teaching-related" propositions are more important for them; front desk counter staff in the Student Centres have different rankings; equity staff etc etc.

I think there is a lack of consistency in terms of how general staff are involved in some activities - eg orientation. Some staff would do nothing and other faculties might be more involved. As a marketing person I am unusual in that I have run our $\mathrm{O}$ camp for a number of years as no-one 
else would do it and I saw the need to do something important but outside my normal duties. I don't think that is usual.

This diversity has been alluded to by other authors, including Conway (2000) who discusses the problematic situation generated by using non-specific term such as "general staff", "non-academic" or "other", to encompass such a large and diverse group of staff. Just as there are typically three classifications used for academic staff — "teaching only", "research only" and "teaching and research" (DEEWR, 2009) - perhaps it is time to be more discerning and descriptive in our nomenclature for general staff, thereby facilitating an improved conceptualisation of the wide range of contributions made by these staff?

\section{Conclusion}

This paper presents the findings of a preliminary study that used the Delphi method to rank a set of behaviours, previously determined to support positive student outcomes (Prebble, et al., 2004), in relation to the contribution of general staff. Middleton (2006) had suggested that general staff were central to these outcomes, and this study investigated this proposal from the perspective of general staff themselves. Overall, the level of agreement was moderate, and a number of propositions were consistently ranked highly over the duration of the study; however, the range of responses also hints at the variety of work undertaken by general staff in support of student outcomes.

As this study was conducted at one site only, the results may not be applicable to all Australian universities. Nevertheless, the methodological issues and the findings presented in this paper are likely to be relevant to many institutions - replication of this study would test this hypothesis and make use of the knowledge of the Delphi method generated by this study. Further in-depth research is planned to develop a rich understanding the work of general staff in Australian universities, particularly in relation to how they contribute to student outcomes. A better understanding and increased appreciation for the diverse contribution of general staff to their university's core activities will enhance the sustainability of Australian universities.

\section{Acknowledgements}

The author gratefully acknowledges the methodological design advice of [name deleted to maintain the integrity of the review process]. This paper forms part of the author's doctoral studies, which are being supervising by [name deleted to maintain the integrity of the review process]. The author would particularly like to thank the general staff at [name deleted to maintain the integrity of the review process], who participated in this study, for making their time and expertise so generously available.

\section{References}

Adams, D. (1998). Examining the fabric of academic life: An analysis of three decades of research on the perceptions of Australian academics about their roles. Higher Education, 36(4), 421-435.

Adler, M., \& Ziglio, E. (1996). Gazing into the oracle: the Delphi method and its application to social policy and public health: Jessica Kingsley Publishers.

Conway, M. (2000). What's in a name?: issues for ATEM and administrators. Journal of Higher Education Policy and Management, 22(2), 199-201.

Delbecq, A., Van de Ven, A. H., \& Gustafson, D. H. (1975). Group techniques for program planning : a guide to nominal group and Delphi processes (A. H. Van de Ven \& D. H. Gustafson, Trans.). Glenview, Ill.: Scott, Foresman. 
Department of Education, Employment, \& Workplace Relations (2008). Students 2007 [full year]: selected higher education statistics, from http://www.dest.gov.au/sectors/higher_education/publications_resources/profiles/Students_2007_ full year.htm

Department of Education, Employment, \& Workplace Relations (2009). Staff 2008: Selected Higher $\begin{array}{lllll}\text { Education } & \text { Statistics } & \text { Retrieved } & 13 & \text { July }\end{array}$ http://www.dest.gov.au/sectors/higher_education/publications resources/statistics/publications_hi gher_education_statistics_collections.htm\#staffpubs

Duffield, C. (1993). The Delphi technique: a comparison of results obtained using two expert panels. International Journal of Nursing Studies, 30(3), 227-237.

Elford, I. C. (1996). Utilization of student outcomes assessment information in institutional decision making. [Research paper]. International Journal of Educational Management, 10(3), 36 - 45.

Fink, A., Kosecoff, J., Chassin, M., \& Brook, R. H. (1984). Consensus methods: characteristics and guidelines for use. American Journal of Public Health, 74(9), 979-983.

Hasson, F., Keeney, S., \& McKenna, H. (2000). Research guidelines for the Delphi survey technique. Journal of Advanced Nursing, 32(4), 1008-1015.

Keeney, S., Hasson, F., \& McKenna, H. (2006). Consulting the oracle: ten lessons from using the Delphi technique in nursing research. Journal of Advanced Nursing, 53(2), 205-212.

Kendall, M. G., \& Gibbons, J. D. (1990). Rank correlation methods (J. D. Gibbons, Trans. 5th edn ed.). London: Edward Arnold.

Marginson, S., \& Considine, M. (2000). The enterprise university: power, governance and reinvention in Australia (M. Considine, Trans.). New York Cambridge University Press.

Middleton, S. (2006). The Divided House in Tertiary: The Importance of Service Departments in Positive Academic Outcomes. Paper presented at the Association for Tertiary Education

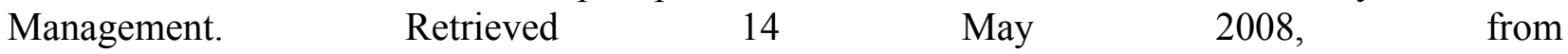
http://pandora.nla.gov.au/pan/10533/20061010/www.temc.org.au/program3.html

Ng, G. C., Heskin, K., \& Sharma, R. (1993). Quality of Student Outcomes: Concepts And Issues Of Measurement. Journal of Institutional Research in Australasia, 2(1). Retrieved from http://www.aair.org.au/jir/Nov93/Ng2.pdf

Okoli, C., \& Pawlowski, S. D. (2004). The Delphi method as a research tool: an example, design considerations and applications. Information \& Management, 42(1), 15-29.

Powell, C. (2003). The Delphi technique: myths and realities. Journal of Advanced Nursing, 41(4), 376382.

Prebble, T., Hargreaves, H., Leach, L., Naidoo, K., Suddaby, G., \& Zepoke, N. (2004). The impact of student support services and academic development programmes on student outcomes in undergraduate tertiary study: A synthesis of the research. Retrieved from http://www.educationcounts.govt.nz/publications/tertiary_education/5519.

Salaway, G., Caruso, J. B., \& Nelson, M. R. (2007). The ECAR study of undergraduate students and information technology, 2007. EDUCAUSE Centre for Applied Research. Retrieved April 11, 2010 from http://www.educause.edu/ECAR/TheECARStudyofUndergraduateStu/161967.

Schmidt, R., Lyytinen, K., Keil, M., \& Cule, P. (2001). Identifying Software Project Risks: An International Delphi Study. Journal of Management Information Systems, 17(4), 5-36.

Schmidt, R. C. (1997). Managing Delphi Surveys Using Nonparametric Statistical Techniques. Decision Sciences, 28(3), 763-774.

Szekeres, J. (2004). The invisible workers. Journal of Higher Education Policy and Management, 26(1), 7-22.

Tait, A. (2000). Planning Student Support for Open and Distance Learning. Open Learning: The Journal of Open and Distance Learning, 15(3), 287 - 299. 